\title{
La Fenomenología de Husserl y Heidegger
}

\section{Husserl and Heidegger's phenomenology}

\section{A fenomenologia de Husserl y Heidegger}

Claudia Alejandra Soto Núñez ${ }^{1}$, Ivonne Esmeralda Vargas Celis ${ }^{2}$

${ }^{1}$ Enfermera educadora, Subdirección de Enfermería Hospital Clinico UC CHRISTUS.

Magister en Enfermería Universidad Católica de Chile. Santiago de Chile.

${ }^{2}$ Magister en Ética Universidad Católica de Lovaina, Bélgica. Docente de la Escuela de Enfermería, Pontificia Universidad Cat . Cómo citar este artículo en edición digital: Soto Núñez, C.A., \& Vargas Celis, I.E. (2017). La Fenomenología de Husserl y Heidegger. Cultura de los Cuidados (Edición digital), 21(48). Recuperado de http://dx.doi.org/10.14198/cuid.2017.48.05 Correspondencia: Hospital Clinico UC CHRISTUS Marcoleta 367, 6º piso Subdireccioón de Enfermería, Santiago, Chile. Correo electrónico: claudias140@gmail.com; ivargase@uc.cl

Recibido: 10/07/2016; Aceptado: 22/02/2017

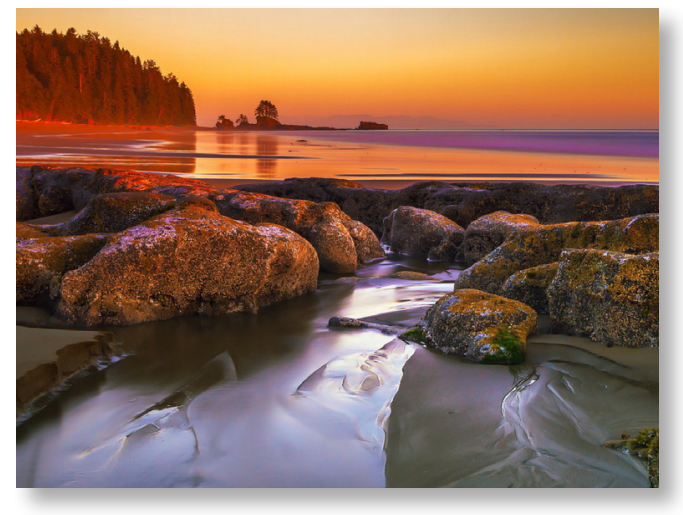

\section{ABSTRACT}

The phenomenological method comes to be an important tool for nursing that allows, among other benefits, to improve the understanding of the human being who is the subject of nursing care. The aim of this article was to know the concepts and postulates of two classical exponents of the phenomenological method, as are the philosophers E. Husserl and M. Heidegger. Method: revision of the phenomenological method used by both authors. Results: Husserl, the founder of phenomenology, basically proposes that through a reflection you can discover that is the invariant present in the experiences of human beings (essence). M. Heidegger, Husserl's disciple, who following the same line, advances and tries to recognize, through language, the Being that is hidden in the middle of his environment (Dasein). Conclusions: the phenomenology comes to be an excellent method of research, very useful for nursing, because it allows unveil the essence of the phenomena associated with care, however, it must be applied with a basis, knowing the theoretical-philosophical postulates that support it.

Key words: Phenomenology, Husserl, Heidegger, Nursing.

\section{RESUMEN}

El método fenomenológico surge para enfermería como una importante herramienta que le permite, entre otros beneficios, mejorar la comprensión del ser humano al cual brinda cuidados. El objetivo de este artículo es conocer los conceptos y postulados de dos exponentes clásicos del método fenomenológico, como son los filósofos Edmund Husserl y Martin Heidegger. Método: revisión del método fenomenológico que utilizan ambos autores. Resultados: E. Husserl, fundador de la fenomenología, propone que a través de la reflexión, se puede descubrir aquello invariable que está presente en las vivencias del ser humano (esencias). M. Heidegger, su discípulo, quien siguiendo la misma línea, avanza e 
intenta reconocer, a través del lenguaje, al Ser que está oculto en medio de su entorno (Dasein). Conclusiones: la fenomenología surge como un excelente método de investigación, muy útil para enfermería, pues permite develar la esencia de los fenómenos asociados al cuidado; sin embargo, éste debe ser aplicado con fundamento, conociendo los postulados teóricos-filosóficos que lo sustentan.

Palabras claves: Fenomenología, Husserl, Heidegger, Enfermería.

\section{RESUMO}

O método fenomenológico surge para a enfermagem, como uma importante ferramenta que lhe permite, entre outros benefícios, melhorar a compreensão do ser humano que lhe brinda cuidados. O objetivo deste artigo é a de conhecer os conceitos e postulados de dois exponentes clássicos do método fenomenológico, como são E. Husserl e M. Heidegger. Método: revisão do método fenomenológico usando ambos os autores. Resultados: E. Husserl, fundador da fenomenologia, propõe que através da reflexão, pode-se descobrir aquela invariante que está presente nas vivencias do ser humano (essências). M. Heidegger, seu discípulo, quem seguindo a mesma linha, avança e tenta reconhecer, através da linguagem, o Ser oculto que esta no meio do seu entorno (Dasein). Conclusões: a fenomenologia emerge como um método de pesquisa excelente, útil para a enfermagem, porque permite revelar a essência dos fenômenos associados cuidados; no entanto, ele deve ser aplicado fundação, conhecer os princípios teóricos e filosóficos que fundamentam.

Palavras chaves: Fenomenologia, Husserl, Heidegger, Enfermagem.

\section{INTRODUCCIÓN}

Desde hace ya tiempo, en el área de la salud, y en especial en la disciplina de Enfermería, ha surgido la necesidad de explorar y comprender las experiencias de vida relacionadas con el cuidado, la enfermedad y la muerte desde la perspectiva de los pacientes y también de sus cuidadores. A partir de ello, ha sido necesario recurrir a metodologías de investigación que permitan profundizar en fenómenos sociales, por lo que el desarrollo de estudios de tipo cualitativo se ha convertido en una herramienta de gran utilidad. Entre ellas, la fenomenología, ha sido un recurso cada vez más utilizado en enfermería, ya que busca sacar a la luz o develar el significado esencial de la experiencia humana, con el fin de comprender mejor a la persona, lo que a su vez podría contribuir a proporcionarle un cuidado más humanizado.

En la actualidad al hablar de fenomenología se tiende a pensar principalmente en la fenomenología de Husserl y sus principales continuadores, pero el llamado movimiento fenomenológico es muy complejo y variado, reconociéndose diferentes exponentes, tales como: Heidegger, Gadamer, Hartman, Sartre, Merleau-Ponty, Ricoeur, entre otros. A lo largo de la historia, también se pueden distinguir distintas fases, dentro de las que destacan la alemana y la francesa (Spiegelberg, 1982). Si bien, la fenomenología nace de la innovación de un método, también aporta otros aspectos metafísicos fundamentales, lo que hace de ella no solo una práctica o una tendencia, sino también una filosofía (Ferrater, 1994). Con el fin de fundamentar el conocimiento teóricofilosófico que abala al método fenomenológico, este artículo revisará a dos exponentes clásicos de la llamada fase alemana: Edmund Husserl (fundador del método) y Martín Hei- 
degger, su discípulo, el cual desarrollará su propia fenomenología con un enfoque más bien existencial (Thévenas, 1966).

\section{DESARROLLO DEL TEMA}

\section{La filosofía de Husserl}

La fenomenología surge a comienzos del siglo XX, como un método científico descriptivo, el cual se establece en conjunto con la psicología apriórica pura o fenomenológica. $\mathrm{Su}$ fundador Edmund Husserl (1859-1938) lo piensa como una respuesta al positivismo científico imperante en la época, ya que este método hipotético-deductivo parte de la premisa, que el mundo se rige por leyes precisas que pueden explicar, predecir y controlar los fenómenos, a partir de lo cual se realizan generalizaciones universales a fenómenos que son multicondicionados (Gonzalez, 2003). Estas afirmaciones no fueron suficientes para conocer la realidad de las cosas, por lo que Husserl irrumpe con un método diferente, que utiliza el análisis reflexivo y que implica una responsabilidad social, a través del estudio de los fenómenos en forma pura ("a las cosas mismas"), no parcializando, ni manipulando al objeto de estudio como lo hacían las ciencias de la época (Thurnher, 1996). Para ello, establece una forma de conocer la realidad, "adentrándose en la esfera interna de las cosas, para captar su verdadero sentido, olvidando lo externo o lo evidente" (Husserl, 1992). Su principal regla es "dejar que las cosas se hagan patentes en su contenido esencial, a través de una mirada intuitiva" (Husserl, 1949).

Un elemento central en la visión husserliana son los fenómenos, que se pueden definir como aquello "que se presenta a la conciencia" por lo que pueden ser percibidos como "pura aparición” (Husserl, 1992). De esta forma, el fenómeno se constituye a través de la percep- ción directa o intuición clara de la conciencia. Lo que Husserl buscaba era lograr establecer una estructura científica para comprender lo subjetivo del pensamiento. Para este filósofo, el vivir psíquico sólo es posible que surja ante la reflexión, pues es por ella que se conocen o "aparecen" estas vivencias subjetivas o fenómenos (Husserl, 1992).

La fenomenología para Husserl es la ciencia que trata de descubrir las estructuras esenciales de la conciencia (Husserl, 1992), y se caracteriza por ir en búsqueda de experiencias originarias (Held, 2009) y exponerlas en su contexto (Thurnher, 1996). Este contexto implica considerar por una parte, un mundo exterior que le da sentido al fenómeno; y por otra parte un mundo interior que da cuenta de cómo es percibida la experiencia como un todo, y desde la perspectiva del que la vive (Thurnher, 1996). Para este filósofo, la fenomenología cobra sentido como método, ya que él consideraba que "las formas de ser, que tienen especial modo de darse, tienen también sus modos en cuanto a las formas de conocerlas" (Thurnher, 1996), es decir que solo llegando a la esencia de las cosas éstas pueden ser conocidas verdaderamente. De ahí que, a partir de las vivencias, se espera llegar a la esencia que permite comprender en profundidad esta experiencia. Según Husserl "toda vivencia que logre una mirada reflexiva, tiene una esencia propia, aprehensible, un contenido susceptible de ser contemplado en su peculiaridad" (Husserl, 1992).

Otro elemento central en la filosofía de Husserl, que sufre diversas modificaciones en la medida que evoluciona su pensamiento, es la conciencia. Ella puede ser entendida de tres formas: a) como total consistencia fenomenológica del yo empírico; b) como percepción interna de las propias vivencias psíquicas; c) 


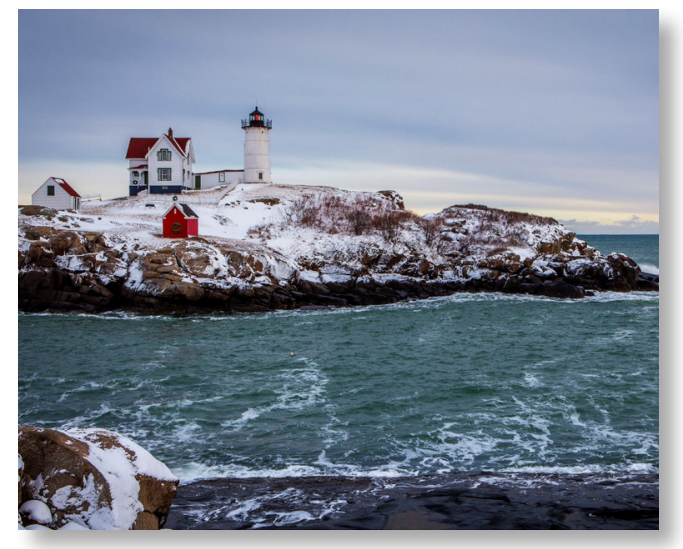

y como el conjunto de actos psíquicos o vivencias intencionales, es decir los fenómenos (Husserl, 1976). Según Husserl, lo característico de la conciencia es su intencionalidad, es decir ella tiene un objeto "intencional" al cual apunta el pensamiento, por lo que está dirigida hacia fuera. La conciencia es "conciencia de"; es un fluir de experiencias que no se detiene (Husserl, 1992), es decir toda audición es audición de algo, toda visión lo es de algo, toda voluntad apunta a algo querido, etc. En la actividad intencional se distinguen dos momentos inseparables en el análisis fenomenológico: el noético, característico de la intuición de las esencias, es decir al cómo se da el objeto; y el noemático, característico del vuelco de la conciencia hacia sí misma, es decir el acto mismo de la conciencia al referirse al objeto (Husserl, 1992).

\section{El Método de Husserl}

El método fenomenológico, según Husserl, establece que el investigador debe partir realizando una reducción histórica de sus experiencias, con el fin de lograr un juicio objetivo y neutral que permita acceder a una conciencia pura (Thurnher, 1996). Esto implica una suspensión (del griego epokhé) del mundo natural, es decir desconectar todas las concepciones filosóficas, teológicas, científi- cas, axiológicas que se tienen del mundo natural y que impiden describir los fenómenos tal "como se manifiestan desnudamente a la conciencia" (Husserl, 1949). Esto es posible, sólo en la medida que se pueda estar libre de cuestionamientos y prejuicios, dejando entre "paréntesis todo lo conocido o experimentado" acerca de lo que se va a analizar, esto es conocido como epojé (Husserl, 1949).

El método continúa con la búsqueda de fenómenos puros (esencias o eidos), a partir de las vivencias captadas por la conciencia, lo que se logra a través de la reducción eidética (Husserl, 1992). Este proceso consiste en "dejar entre paréntesis todo lo variable o relativo" y capturar sólo lo que permanece estable, llegando al núcleo invariante de la situación (Thurnher, 1996), es decir, a su esencia. Las esencias son dadas a la intuición fenomenológica a partir del encuentro de múltiples miradas o puntos de vista (García, 2000), la cual se convierte en una aprehensión de "unidades ideales significativas" (de sentido u objetos de sentido), o de universalidades (Husserl, 1992, Lamber, 2006).

Lo anterior da paso a otro momento del método la reducción fenomenológica trascendental, donde se hace referencia al ámbito de las operaciones constitutivas de la conciencia, que se hacen presente en las reducciones de las vivencias. Con ello se espera "abrir la conciencia” para llegar a una verdadera descripción fenomenológica, que lleva a una conciencia absoluta (Thurnher, 1996). Esto implica, poner entre paréntesis la conciencia de la existencia misma, es decir la "creencia que el ser considera a las cosas como perteneciendo a la conexión de un mundo que existe independiente de nuestra conciencia" (Thurnher, 1996). Por lo tanto, es necesario poner entre paréntesis el yo fáctico mundano para abrirse a través de 
una actitud reflexiva, a una "perspectiva de actos intencionales de una conciencia pura general" (Thurnher, 1996). Dicho de otra forma, cuando se abandona el mundo conocido o cotidiano, donde se realizan las actividades prácticas y teóricas, el mundo pre-dado, se puede pasar al mundo de la conciencia (Husserl, 1992), pues la conciencia está constitutivamente dirigida al mundo, y el mundo existe ante una conciencia.

En forma practica, para el investigador que desea basar su estudio fenomenológico en los postulados de Husserl, debe comenzar realizando el proceso de reducción fenomenológica antes de comenzar con las entrevistas, poniendo de manifiesto todas las ideas y creencias preconcebidas que tiene sobre el fenómeno que va estudiar, con el fin de tener claridad de cuáles son esas percepciones y dejarlas a un lado o entre paréntesis, para así no contaminar aquello que surgirá de las experiencias vividas por los entrevistados. Esto también implica partir el estudio con un conocimiento somero sobre el fenómeno, y una vez obtenidos los resultados finales, el investigador podrá revisar la literatura y contrastar los resultados con otras investigaciones. Este proceso inicial de reconocimiento, se debe realizar durante todo el proceso de análisis de las entrevistas, y se conoce como bracketing (Streubert y Carpenter, 2007).

Otro aspecto fundamental, al que aspira Husserl en su método es poder llegar a captar la esencia del fenómeno. Esto es posible en la investigación fenomenológica, a través de la entrevista en profundidad, la cual se caracteriza por tener una mínima intervención del entrevistador, y a continuación con la lectura y relectura de estas entrevistas, con el fin de compenetrarse con los relatos e ir captando aquello invariable que se va repitiendo, aun- que se revele de diferentes maneras (Streubert y Carpenter, 2007). Para lograr la confiabilidad de la esencia captada, se recomienda que este proceso sea realizado por uno o más coinvestigadores en forma independiente, para luego contrastar los resultados.

\section{La filosofía de Heidegger}

Martin Heidegger, filósofo y discípulo de Husserl, desarrolla una filosofía existencial a partir de la cual contribuye notablemente al desarrollo de la fenomenología. A partir de ella establece al ser en el mundo socio-histórico, donde la dimensión fundamental de toda conciencia humana es histórica y sociocultural, la que se expresa por medio del lenguaje (Ferrater, 1994). Para Heidegger, a partir de la fenomenología se logra captar aquello estable o permanente desde lo cambiante en el mundo. Esto no se logra a partir de preguntas sin sentido, sino a través de escuchar la expresión de las vivencias que no se muestran a simple vista y que se deben descubrir (Bertorello, 2006). Como eje central de su filosofía existencial, Heidegger se pregunta por el ser: ¿cuál es el sentido del ser?, ¿qué significa el ser?, y su método para descubrirlo es a través de la fenomenología hermenéutica del ser o Dasein.

Heidegger establece que el ser sólo puede ser interpretado desde una mirada ontológica que logra "una reconstrucción de la mirada en el ser ahí, que busca lo oculto detrás de lo manifiesto, y lo no comprendido enfáticamente" (Horneffer, 2009). El estar ahí es su propia posibilidad o esencia, por eso su naturaleza consiste en su existencia (Ferrater, 1994). En otras palabras, no es entorno el que le da sentido a la existencia del ser, sino por el contrario.

Para Heidegger, la fenomenología es un importante medio que logra "conservar las vivencias de la conciencia como su ámbito 
temático" (Heidegger, 2003), quedando establecido que los fenómenos normalmente no se dan o donan en la vida cotidiana, hasta que existe una preocupación por develarlos (Thurnher, 1996). Para Heidegger, la manera como se presenta o es interpretado el ser, determina los múltiples modos del ser, es decir los entes (Thurnher, 1996). El ser está oculto o encubierto en el ente, es decir participa del ente y la idea central es poder obtener al ser que hay en él para descubrirlo de verdad (Heidegger, 2003). En otras palabras, el fenómeno es el ser que debe sacarse a la luz, ya que se encuentra oculto en medio de otros fenómenos más superficiales que llaman primeramente la atención (De la Maza, 2005). Esto sólo es posible a partir de la fenomenología hermenéutica, la cual transforma una comprensión distorsionada u oculta del ser, en una comprensión del ser en sí mismo (Wandelfen, 1997).

\section{El método de Heidegger}

El método fenomenológico para Heidegger puede dividirse en tres partes: destrucción, reducción y construcción fenomenológica. En la destrucción fenomenológica, se deben hacer consientes los prejuicios y posturas frente al mundo (Thurnher, 1996). Él postula que no es posible negar lo conocido sobre lo que se está examinando, tal como lo indicara Husserl, ya que los conceptos asociados son tan potentes, que de algún modo se manifiestan inadvertidamente (De la Maza, 2005). Por el contrario, Heidegger llama a hacer un reconocimiento e identificación de tendencias, en estos pensamientos iniciales, $y$ a tener presente que pueden incidir, positiva o negativamente, en la descripción del ser (Thurnher, 1996). En la segunda etapa llamada reducción fenomenológica, viene un proceso de escucha del ente ante lo "no dicho", teniendo presente que el ser utiliza los entes y su entorno con tal familiaridad, que en su relato solo es posible percibir la esencia, rompiendo esta familiaridad y adentrándose en él (Horneffer, 2009). Si se aprende a escuchar en el ente lo no dicho, es posible que éste se dé o se done (Horneffer, 2009).

Finalmente, en la tercera etapa de construcción fenomenológica, se espera lograr la comprensión del fenómeno donado. Para Heidegger, la donación no es un proceso inmediato y libre de supuestos, sino que debe ser analizado y criticado, a través de un proceso de pre-concepción hermenéutica (Thurnher, 1996, Horneffer, 2009). A partir de este proceso, es posible captar lo que está oculto, capturando el mensaje que hay en el lenguaje, hasta llegar a su comprensión (De la Maza, 2005), o en el mejor de los casos, intuiciones esenciales (Horneffer, 2009).

El Dasein, es decir este ser que se dona, no sólo es apariencia, sino que tiene múltiples constituciones, ya que está en el mundo (Thurnher, 1996), "familiarizado con lo significativo que lo contiene y que lo atrapa”. Es el ser que logra abrirse al mundo en su contexto temporal (Thurnher, 1996), "un yo histórico que vive aquí y ahora en el mundo de sus preocupaciones" (Bertorello, 2006), el cual es posible comprender a través de la interpretación de sus manifestaciones, las que se deben describir, tal y como se presentan.

Para ser consecuentes y rigurosos con el método de Heidegger, el investigador debe comenzar con un reconocimiento acucioso de sus percepciones y juicios sobre el fenómeno a estudiar, pero a diferencia de Husserl, será con la idea que estas experiencias van a contribuir a descubrir y comprender el fenómeno o ente que está encubierto. De esta manera, en las entrevistas en profundidad, el instrumento de análisis será el propio investigador, el cual a 
través del lenguaje verbal y no verbal, será capaz de ir comprendiendo lo que quiere decir el entrevistado, en cuanto a si mismo. Finalmente en este método, se debe tener presente que el ser que se devela, está inserto en un entorno, en un tiempo y en un espacio que lo determina, pero que a su vez, él define y modifica con su sola presencia.

$\mathrm{Si}$ tomamos como ejemplo a la enfermera(o) integra y diligente que día a día atiende a su paciente moribundo, podríamos descubrir al ser que está "detrás" de esa profesional de la salud, que siente temor y tristeza, pero que por el contrario se demuestra fuerte y eficiente para entregar sostén y sentido a un paciente al cual se le va la vida. Esa es su experiencia vital, su Dasein, que le otorga la posibilidad de relacionarse con el que cuida. En la medida que ella reconoce su existencia y se conoce a sí misma, podrá identifica las necesidades del otro y ayudarlo (Ramírez \& Cárdenas \& Rodríguez, 2015).

Las diferencias y semejanzas entre estos dos padres de la fenomenología, se entienden mejor visualizándolas mediante un cuadro comparativo (Cuadro I).

\section{Cuadro I. Cuadro comparativo entre la fenomenología de Husserl y de Heidegger}

\begin{tabular}{|c|c|c|}
\hline & HUSSERL & HEIDEGGER \\
\hline Tipo & $\begin{array}{l}\text { Fenomenológica eidética } \\
\text { lógica. }\end{array}$ & $\begin{array}{l}\text { Fenomenología hermenéutica } \\
\text { (interpretativo) del Dasein. }\end{array}$ \\
\hline $\begin{array}{l}\text { Enfoque } \\
\text { filosófico }\end{array}$ & $\begin{array}{l}\text { Epistemológico. } \\
\text { De la naturaleza } \\
\text { conocimiento. } \\
\text { Énfasis en el regreso a la } \\
\text { intuición reflexiva } \\
\text { escribir y yara } \\
\text { experiencia tal como se vive. } \\
\text { Se constituye en } \\
\text { conciencia. }\end{array}$ & $\begin{array}{l}\text { Existencial (Ontológico). } \\
\text { De la naturaleza de la existencia. } \\
\text { Ser en el mundo socio-histórico, } \\
\text { donde la dimensión fundamental } \\
\text { de toda conciencia humana es } \\
\text { histórica y sociocultural. }\end{array}$ \\
\hline $\begin{array}{l}\text { Principal } \\
\text { Característica }\end{array}$ & $\begin{array}{l}\text { Conciencia intencional. } \\
\text { Epojé (poner entre } \\
\text { paréntesis). } \\
\text { Eidos o esencias. } \\
\text { Reflexividad de la conciencia. }\end{array}$ & $\begin{array}{l}\text { Dasein el Dasein (Da-sein) ser } \\
\text { ahí/estar en el mundo. } \\
\text { Analítica existenciaria del modo } \\
\text { por el cual se accede a la pregunta } \\
\text { por el sentido del ser. }\end{array}$ \\
\hline Lenguaje & $\begin{array}{l}\text { Descriptivo. } \\
\text { Su propósito es que la } \\
\text { experiencia original sea } \\
\text { evidente por medio de la } \\
\text { intuición clara. }\end{array}$ & $\begin{array}{l}\text { Manifestación del ser. } \\
\text { Hermenéutica: conocer } \\
\text { interpretar. }\end{array}$ \\
\hline Fenómeno & $\begin{array}{l}\text { Se constituye a través de la } \\
\text { percepción directa o intuición } \\
\text { clara. }\end{array}$ & $\begin{array}{l}\text { "Estar-en-el-mundo" es "Ser-en- } \\
\text { el-mundo". }\end{array}$ \\
\hline Pregunta & Cómo conozco el fenómeno? & $\begin{array}{l}\text { ¿Cuál es el sentido del ser? ¿Qué } \\
\text { significa ser?. }\end{array}$ \\
\hline
\end{tabular}




\section{CONCLUSIONES}

Husserl, como fundador del método fenomenológico, se presenta como un provocador para la época de las ciencias exactas, al plantear un método riguroso de estudio, que pretende descubrir aquello invariante en las vivencias del ser humano. Heidegger, siguiendo la misma línea, avanza un poco más allá, e intenta reconocer al ser oculto que está en medio de su entorno. Ambos exponentes de esta filosofía, se esmeraron por perfeccionar la comprensión del ser humano y del mundo, desde una mirada distinta, sin pre concepciones y profundamente holística.

El conocimiento teórico filosófico que abala al método fenomenológico, permite al investigador un trabajo riguroso en la interpretación fiel y acabada de las vivencias obtenidas en su estudio. Develar el real significado del sufrimiento, el sentido de la experiencia vivida tanto en una persona enferma o que se ve enfrentada a la muerte, entre otras temáticas; demuestra un interés genuino y respetuoso por su existencia y fragilidad. Enfermería, en su que hacer clínico, se ve continuamente enfrentada a este tipo de situaciones, muchas veces desconocidas o temidas, por lo que la fenomenología surge como un excelente método de investigación, que debe ser aplicado con fundamento, conociendo la filosofía que la sustenta.

\section{BIBLIOGRAFÍA}

- Bertorello, A. (2006). La filosofía de Heidegger como una teoría del sujeto de la enunciación. Revista lenguaje sujeto de discurso, (3), 36-44.

- De la Maza, L. (2005). Fundamentos de la filosofía hermenéutica: Heidegger y Gadamer. Teología y Vida, 56, 122-138. Disponible en http://www. scielo.cl/scielo.php? script $=$ sci arttext $\&$ pid $=$ S0049$\underline{34492005000100006 \& \operatorname{lng}=\text { es\&nrm }=\text { iso }}$

- Gonzalez, A. (2003). Los paradigmas de investigación en las ciencias sociales. Revista de la Universidad Central “Marta Abréu de Las Villas". Islas, 45(138) 125-135.
- Ferrater, J. (1994). Diccionario de Filosofía. Vol. 2. Barcelona: Ariel.

- Garcia, A. (2000). Introducción a la fenomenología de Edmund Husserl. Revista de ciencias humanas, 22.

Disponible en http://www.utp.edu.co/ chumanas/revistas/revistas/rev22/garcia.htm

- Held, K. (2009). Fenomenología del 'tiempo propio' en Husserl y Heidegger. La lámpara de Diógenes, 10(18-19), 22-29.

- Heidegger, M. (2003). Mi camino en la fenomenología. En: Tiempo y ser. Madrid: Tecnos, 1-7.

- Horneffer, R. (2009). En torno a la fenomenología de Heidegger. Revista filosofía, 41(124), 117-128.

- Husserl, E. (1949). La conciencia y la realidad natural. En: Ideas relativas a una fenomenología pura y una filosofía fenomenológica. Buenos Aires: Fondo de Cultura Económica, 74-90.

- Husserl, E. (1976). Investigaciones lógicas. Madrid: Alian-za.

- Husserl, E. (1992). El articulo “fenomenología”. En: Invi-tación a la fenomenología. Barcelona: Paidós, 35-73.

- Lamber, C. (2006). Edmund Husserl: la idea de la fenomenología. Teología y Vida, Vol. XLVII, 517 - 529.

- Ramírez, M., Cárdenas, M., Rodríguez (2015). El Dasein de los cuidados desde la fenomenología hermenéutica de Martín Heidegger. Enfermería universitaria, 12(3), 143-151.

- Spiegelbert, H. (1982). The phenomenological movement: a historical introduction. The Hague: M.Nijhoff.

- Streubert H., Carpenter D. (2007). Qualitative research in Nursing: Advancing the humanistic imperative. $4^{\circ}$ ed. Philadelphia: Lippincott Williams \& Wilkins.

- Thévenaz, P. (1966). De Husserl à Merleau-Ponty: qu'est-ce que la phénoménologie? Neuchatel: Suisse Editions de la Baconnière.

- Thurnher, R. (1996). “¡A las cosas mismas!” Acerca de la significación de la máxima fenomenología fundamental en Husserl y Heidegger. Seminarios de filosofía, 9, 24-44. 